Umur Hatipoğlu, MD

Department of Critical Care Medicine

Respiratory Institute, Cleveland Clinic

\title{
Can multiple ARDS patients be ventilated with a single ventilator?
}

ACED with A SURGE in demand for mechan4 ical ventilators and fewer machines than patients who need them, hospitals are considering ventilating more than 1 patient with a single ventilator (multiplex ventilation). First reported as technically feasible in a test lung model, ${ }^{1}$ the technique was anecdotally used in humans after the 2017 Las Vegas shooting. ${ }^{2}$

More COVID-19 Curbside Consults: www.ccjm.org

In response to the COVID-19 pandemic, several methods of providing mechanical ventilation to 2 to 9 patients have been posted on the Internet. Almost all of them involve diverting flow with off-the-shelf respiratory $\mathrm{Y}$ connectors applied at the inspiratory and expiratory ports of the ventilator (Figure 1). Valves, typically water valves, are repurposed for regulating gas flow (Figure 2). Some of these techniques employ 3-D printed devices that divide flow.

Mechanical ventilation is a life-support intervention. In acute respiratory distress syndrome (ARDS), it has several distinct goals, including maintaining low tidal volume ventilation, controlling positive end-expiratory pressure to improve oxygenation, removing enough carbon dioxide, and providing enough oxygen gas concentration to avoid hypoxemia.

To what extent can multiplex ventilation achieve these goals in individual patients with ARDS? What are the critical problems that need to be addressed for it to be practical and safe?

\section{MECHANISTIC ISSUES}

From a mechanistic standpoint, 3 critical issues need to be addressed to minimize risk to doi:10.3949/ccjm.87a.20043

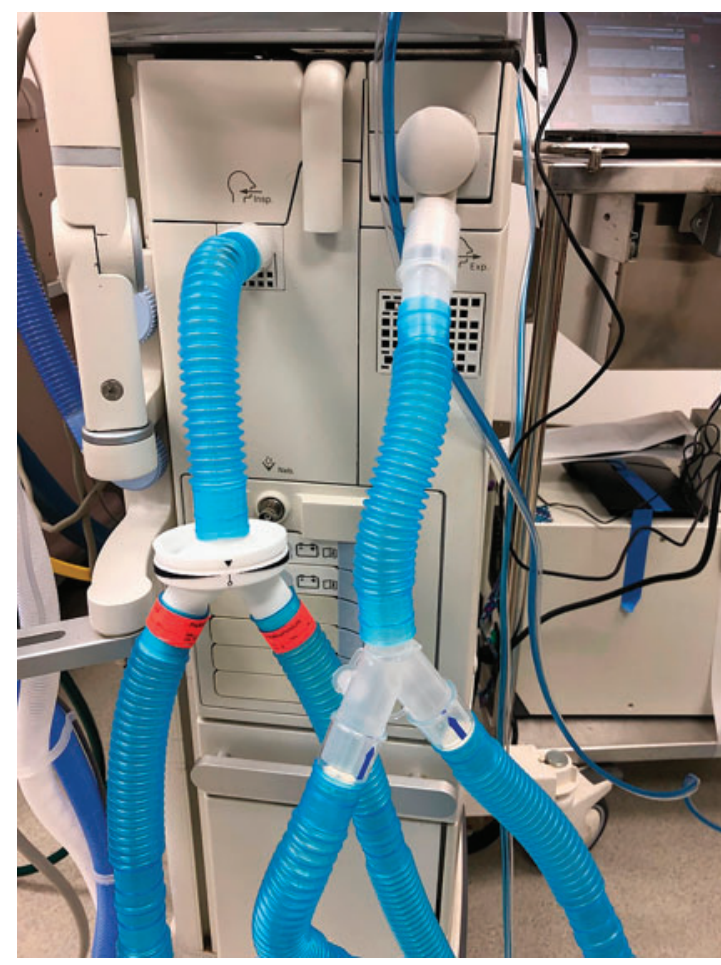

Figure 1. Inspiratory and expiratory inputs to ventilator, with $Y$ connectors.

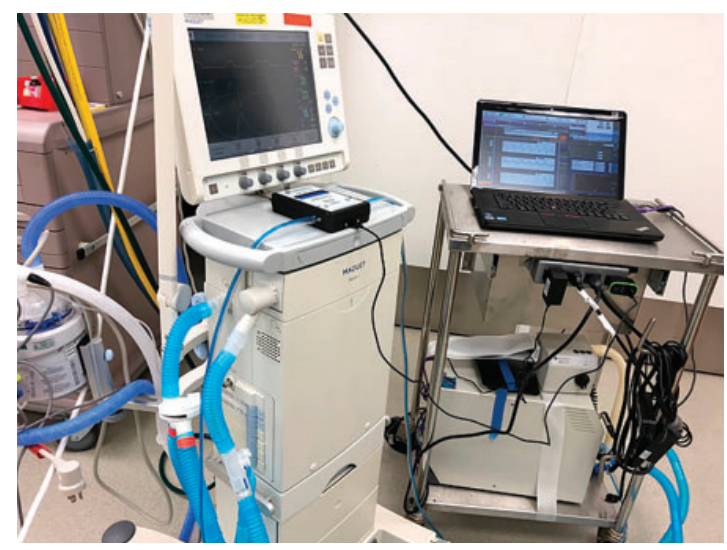

Figure 2. Ventilator setup with volume monitor.
With more patients than machines, multiplex ventilation is a reasonable last resort to prevent certain death 
patients on multiplex ventilation:

Partitioning the volume delivered by the ventilator to each patient. The volumes and pressures registered by the ventilator represent the aggregate volume and total pressure delivered to both patients. Without any intervention, partitioning of volumes between the patients would depend on the respiratory system mechanics of each patient. Therefore, the first issue is partitioning inspiratory flow from the ventilator individually between patients, ensuring safe volume delivery to each.

Measuring tidal volume delivered to each patient.

Providing individualized positive endexpiratory pressure.

These mechanistic issues are less relevant if the ventilator partners are evenly matched for respiratory mechanics, but if their disease courses take different directions, the best-case scenario in matching can turn into the worst.

\section{CLINICAL ISSUES}

There are several important issues concerning clinical management in multiplex ventilation:

Patients need to receive neuromuscular

Several mechanistic and clinical issues need to be addressed blockade. If this is not done, the individual triggering efforts by patients could create significant patient-ventilator dyssynchrony, hyper- or hypoventilation, and potential exchange of gases between patients. However, neuromuscular blockade is associated with prolonged mechanical ventilation.

Gradual withdrawal of support (weaning from mechanical ventilation) is impossible for an individual patient, as it would affect the partner.

There is no known way to provide different oxygen concentrations to the individual patients. This means that the ventilator should be set at the minimum oxygen concentration that would achieve adequate saturation in the patient with worse oxygenation. This could potentially be too high for the patient next door.

Ventilator-related complications such as mucus plugging or pneumothorax in a single patient could go undetected due to lack of individual alarms. During this time, each patient could get lower tidal volumes if on volume-control ventilation, or 1 patient could get critically low tidal volumes in pressurecontrol ventilation.

Such pitfalls increase risk of harm to a patient who was being ventilated by his or her own machine and now has to share. Ideally, there would happen to be 2 patients who are intubated at the same time with 1 ventilator available, but chances are that alternative scenarios occur when one simply runs out of ventilators. Consequently, ethical concerns arise.

Infection risk. Although the risk of infection can be mitigated with appropriate filter placement in multiplex ventilation circuits, pairs of patients should be chosen so that both either have or do not have COVID-19.

Given these risks, several professional societies have issued a joint statement cautioning against the use of multiplex ventilation with currently available equipment. ${ }^{3}$

\section{A REASONABLE LAST RESORT}

Multiplex ventilation is a reasonable last resort. There are no human data available to inform its practice, and therefore its routine use cannot be endorsed. However, COVID-19 has stretched resources to the limit and forced clinicians to consider multiplex ventilation to prevent certain death.

Clearly written protocols and trained staff are essential for implementation, with the goal of temporary support until individual ventilators are available. Two hospitals in New York City had to issue protocols for implementation of multiplex ventilation. One of these protocols has been cited by the US Public Health Service Commissioned Corps. ${ }^{4}$

Individual monitoring with the necessary alarm controls and ability to change ventilator parameters to suit individual needs are surmountable technical obstacles. The ventilator industry has to consider design features to enable safe multiplex ventilation for future pandemics. 


\section{HATIPOĞLU}

\section{REFERENCES}

1. Neyman G, Irvin CB. A single ventilator for multiple simulated patients to meet disaster surge. Acad Emerg Med 2006; 13(11):1246-1249. doi:10.1197/j.aem.2006.05.009

2. Lake CK. A day like no other. A case study of the Las Vegas mass shooting. Nevada Hospital Association. https:// drive.google.com/file/d/1CxbLHiWJwL9ZRbWddbaPv15N N6YiNFEc/view. Accessed April 6, 2020

3. The Society of Critical Care Medicine (SCCM), American Association for Respiratory Care, American Society of Anesthesiologists, Anesthesia Patient Safety Foundation, American Association of Critical Care
Nurses, American College of Chest Physicians. American joint statement on multiple patients per ventilator. March 26, 2020. https://www.aarc.org/wp-content/ uploads/2020/03/032620-COVID-19-press-release.pdf. Accessed April 6, 2020

4. U.S. Public Health Service Commissioned Corps. Optimizing ventilator use during the COVID-19 pandemic. March 31, 2020. https://www.hhs.gov/sites/default/files/optimizing-ventilator-use-during-covid19-pandemic.pdf

ADDRESS: Umur Hatipoğlu, MD, Respiratory Therapy, Respiratory Institute, A90 Cleveland Clinic, 9500 Euclid Avenue, Cleveland, OH 44195; hatipou@ccf.org

COVID-19: Perspectives of 3 generations

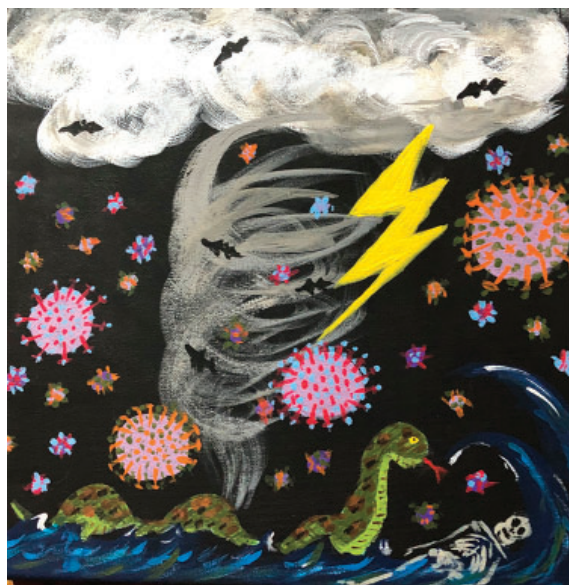

A Tsunami of Death and Destruction Kristin Highland, pulmonary and critical care physician and mother, age 51

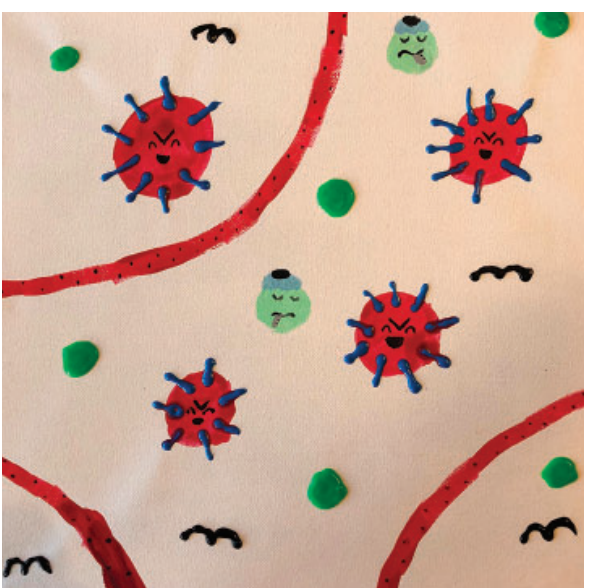

Viral Particles in the Blood Stream and Tissues. The Red Blood Cells Are Green and Glow in the Dark. The White Blood Cells That Fend Off Infection Are All Sick. Small Bats Are Flying Around Rosemary Highland, 5th-grade student and daughter, age 11

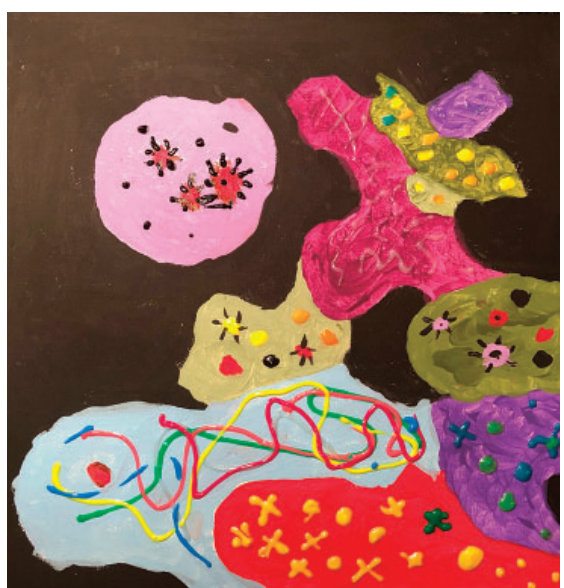

The Virus Is Mutating, and There Are Various Stages of Inflammation

Martha Highland, mother and grandmother, age 78 